\title{
Seasonal Incidence of Galleria mellonella in Stored Wax Combs and its Correlation with Weather Parameters
}

\author{
Lalita and Yogesh Kumar \\ Department of Entomology, CCS Haryana Agricultural University, Hisar-125 004, India \\ E-mail: Ialitapanwar17@gmail.com
}

\begin{abstract}
The investigation was carried out at CCS Haryana Agricultural University, Hisar for two years i.e. 2016 and 2017 during June to October. During June, population of larvae of Galleria mellonella larvae started increasing till September in both years.. However, during October, the number decreased. This pattern of seasonal incidence was same when eight, nine and ten combs were stored in a hive body (chamber). The number of larvae recorded per comb was highest when ten combs were stored in a hive body followed by 9,8 and 7 combs. During both the years, pupal and adult population in stored combs also increased with from June to September while in October, the cumulative number of pupae and adults decreased. Correlation studies between number of wax moth population (larvae, pupae and adults) in stored combs related to different frame strength with the different weather parameters showed significant positive correlation with maximum $\mathrm{RH}$ and maximum temperature, whereas no significant correlation existed with min. temperature, min. $\mathrm{RH}$ and number of bright sunshine hours.
\end{abstract}

Keywords: Fumigation, Wax moth, Galleria mellonella

Galleria mellonella is a cosmopolitan, devastating and economically important pest causing serious economic damage to honey bee combs and stored bees wax during storage (Gillard 2009, Ellis and Hayes 2009). Wax moths are nocturnal pest flying at night and hide in dark places during the day time and stored combs are ideal places for breeding of moths. Larva is the most destructive stage of the pest. Besides damaging wax comb, larvae consume bees wax, destroying frames and wooden parts in the hive (Ellis et al 2013). The population of wax moth fluctuates according to weather conditions. Maximum infestation of wax moth was recorded during July to September, generally in the brood frames and occasionally in the super frames. In Sudan, Fathy et al (2017) reported that the high infestation of larvae of the GWM and LWM in storage in May, June and July but pupae and adult of GWM in storage during June to September. Stored combs without fumigation reported maximum infestation of wax moth (Kumari and Jha 2013). Therefore, the present work aimed to study incidence of the Galleria in the storage combs.

\section{MATERIAL AND METHODS}

All the combs were used for experimental purpose were initially fumigated with aluminium phosphide (Celphos) tablets. For this purpose, 9 combs were placed in each chamber and a stack of 4 chambers were made. The fumigated combs were used for experimental purpose after 7 days. For recording seasonal incidence of greater wax moth in stored combs 7, 8, 9 and 10 empty combs (without bees) were stored in hive bodies which were placed on bottom board and covered with inner cover. Each of the chambers with frames was made air tight by mud plastering to seal cracks and crevices. After making the chamber airtight, it was covered with top cover. Fortnightly observations were recorded on number of larvae, pupae and adults in experimental stored combs. Temperature and relative humidity (\%) data were collected from observatory of Department of Agriculture Meteorology, CCS Haryana Agricultural University, Hisar. Each treatment was replicated five times from June to October.

\section{RESULTS AND DISCUSSION}

Seasonal incidence of greater wax moth (G. mellonella) larvae: During June, 2016 the number of larvae recorded per comb were 1.40 increased till September (29.60 on $30^{\text {th }}$ September). However, during October, the number decreased to 13.60 on $30^{\text {th }}$ October. This pattern of seasonal incidence was same when eight, nine and ten combs were stored in a hive body (chamber) during both years. On $30^{\text {th }}$ October, the number of larvae recorded per comb was highest (13.60) when ten combs were stored in a hive body followed by nine combs in 2017. Mandal and Vishwakarma (2016) reported highest number of egg clusters and larvae of G. mellonella infesting $A$. mellifera combs during $37^{\text {th }}$ and $38^{\text {th }}$ 
standard week during storage. Fathy et al (2017) reported the abundance of GWM in different months in 2014 highest population activity of the larvae/ colony were in June (36) followed by May (26).

Pupal population of greater wax moth (G. mellonella): During June, 2016 the number of pupae recorded per comb were 0.20 increased till September $\left(26.80\right.$ on $30^{\text {th }}$ September). However, during October, the number decreased to 9.40 on $30^{\text {th }}$ October. This pattern of seasonal incidence was same when eight, nine and ten combs were stored in hive body during both years. On $30^{\text {th }}$ October, the number of larvae recorded per comb was highest (12.80) when ten combs were stored in a hive body followed by nine, eight comb and seven comb stored in 2017. Mandal and Vishwakarma (2016) indicated that the highest number of pupae (23.67) of G. mellonella infesting A. mellifera combs was observed during $37^{\text {th }}$ and $38^{\text {th }}$ standard week in changing environmental conditions. The highest number of GWM pupae (89.67) was observed in September.

Adult population of greater wax moth (G. mellonella): During June, 2016 the number of adult recorded per comb were 0.00 increased till September $\left(26.00\right.$ on $30^{\text {th }}$ September). However, during October, the number decreased to 8.20 on $30^{\text {th }}$ October. This pattern of seasonal incidence was same when eight, nine and ten combs were stored in hive body during both years. On $30^{\text {th }}$ October, the number of adults per comb was highest (11.00) when ten combs were stored in a hive body followed by nine, eight comb and seven comb stored in 2017. Mandal and Vishwakarma (2016) indicated that the highest number of adult (8.00) of G. mellonella infesting $A$. mellifera combs was during $37^{\text {th }}$ and $38^{\text {th }}$ standard week during storage. The

Table 1. Seasonal incidence of Greater wax moth (G. mellonella) larvae during June to October in stored combs during 2016 and 2017

\begin{tabular}{|c|c|c|c|c|c|c|c|c|c|c|c|}
\hline \multirow{3}{*}{$\begin{array}{l}\text { Number of combs in } \\
\text { hive body }\end{array}$} & \multicolumn{10}{|c|}{ Cumulative number of larvae/comb during different months of 2016} & \multirow[t]{3}{*}{ Mean } \\
\hline & \multicolumn{2}{|c|}{ June } & \multicolumn{2}{|c|}{ July } & \multicolumn{2}{|c|}{ August } & \multicolumn{2}{|c|}{ September } & \multicolumn{2}{|c|}{ October } & \\
\hline & 15.6.16 & 30.6.16 & 15.7.16 & 30.7 .16 & 15.8.16 & 30.8 .16 & 15.9.16 & 30.9 .16 & 15.10.16 & 30.10 .16 & \\
\hline Seven & $\begin{array}{c}1.40 \\
(1.54)\end{array}$ & $\begin{array}{c}3.00 \\
(1.98)\end{array}$ & $\begin{array}{c}5.60 \\
(2.54)\end{array}$ & $\begin{array}{l}12.20 \\
(3.61)\end{array}$ & $\begin{array}{l}19.40 \\
(4.51)\end{array}$ & $\begin{array}{l}24.00 \\
(4.98)\end{array}$ & $\begin{array}{l}27.20 \\
(5.29)\end{array}$ & $\begin{array}{l}29.60 \\
(5.50)\end{array}$ & $\begin{array}{l}20.00 \\
(4.58)\end{array}$ & $\begin{array}{l}13.60 \\
(3.81)\end{array}$ & $\begin{array}{l}15.60 \\
(3.83)\end{array}$ \\
\hline Eight & $\begin{array}{c}1.80 \\
(1.65)\end{array}$ & $\begin{array}{l}3.80 \\
(2.17)\end{array}$ & $\begin{array}{l}6.20 \\
(2.66)\end{array}$ & $\begin{array}{l}15.00 \\
(3.99)\end{array}$ & $\begin{array}{l}20.40 \\
(4.60)\end{array}$ & $\begin{array}{l}25.20 \\
(5.11)\end{array}$ & $\begin{array}{l}28.40 \\
(5.41)\end{array}$ & $\begin{array}{c}31.00 \\
(5.6)\end{array}$ & $\begin{array}{l}24.40 \\
(5.03)\end{array}$ & $\begin{array}{l}13.80 \\
(3.84)\end{array}$ & $\begin{array}{l}17.00 \\
(4.01)\end{array}$ \\
\hline Nine & $\begin{array}{c}2.40 \\
(1.82)\end{array}$ & $\begin{array}{c}4.00 \\
(2.23)\end{array}$ & $\begin{array}{c}7.60 \\
(2.89)\end{array}$ & $\begin{array}{l}16.60 \\
(4.19)\end{array}$ & $\begin{array}{l}23.00 \\
(4.88)\end{array}$ & $\begin{array}{l}26.60 \\
(5.23)\end{array}$ & $\begin{array}{l}33.20 \\
(5.83)\end{array}$ & $\begin{array}{l}35.40 \\
(6.03)\end{array}$ & $\begin{array}{l}24.80 \\
(5.06)\end{array}$ & $\begin{array}{l}16.60 \\
(4.16)\end{array}$ & $\begin{array}{l}19.02 \\
(4.23)\end{array}$ \\
\hline Ten & $\begin{array}{c}2.80 \\
(1.92)\end{array}$ & $\begin{array}{c}4.60 \\
(2.36)\end{array}$ & $\begin{array}{c}9.80 \\
(3.24)\end{array}$ & $\begin{array}{l}18.20 \\
(4.37)\end{array}$ & $\begin{array}{l}25.80 \\
(5.17)\end{array}$ & $\begin{array}{l}29.00 \\
(5.47)\end{array}$ & $\begin{array}{l}32.80 \\
(5.80)\end{array}$ & $\begin{array}{l}36.40 \\
(6.11)\end{array}$ & $\begin{array}{l}25.60 \\
(5.15)\end{array}$ & $\begin{array}{l}16.80 \\
(4.21)\end{array}$ & $\begin{array}{l}20.18 \\
(4.38)\end{array}$ \\
\hline Mean & $\begin{array}{c}2.10 \\
(1.73)\end{array}$ & $\begin{array}{c}3.85 \\
(2.19)\end{array}$ & $\begin{array}{c}7.30 \\
(2.83)\end{array}$ & $\begin{array}{l}15.50 \\
(4.04)\end{array}$ & $\begin{array}{l}22.15 \\
(4.79)\end{array}$ & $\begin{array}{l}26.20 \\
(5.20)\end{array}$ & $\begin{array}{l}30.40 \\
(5.58)\end{array}$ & $\begin{array}{l}33.10 \\
(5.82)\end{array}$ & $\begin{array}{l}23.70 \\
(4.95)\end{array}$ & $\begin{array}{r}15.20 \\
(4.01)\end{array}$ & - \\
\hline Factors & & & & C.D. & & & $\mathrm{SE}(\mathrm{d})$ & & & $\mathrm{SE}(\mathrm{m})$ & \\
\hline Month & & & & 0.11 & & & 0.05 & & & 0.04 & \\
\hline Combs & & & & 0.18 & & & 0.09 & & & 0.06 & \\
\hline \multirow[t]{2}{*}{ Month $\times$ Combs } & & & & 0.36 & & & 0.18 & & & 0.12 & \\
\hline & \multicolumn{10}{|c|}{ Cumulative number of larvae/comb during different months of 2017} & Mean \\
\hline Seven & $\begin{array}{c}1.80 \\
(1.66)\end{array}$ & $\begin{array}{c}3.20 \\
(2.04)\end{array}$ & $\begin{array}{c}6.00 \\
(2.61)\end{array}$ & $\begin{array}{l}12.60 \\
(3.66)\end{array}$ & $\begin{array}{l}19.80 \\
(4.55)\end{array}$ & $\begin{array}{l}25.00 \\
(5.08)\end{array}$ & $\begin{array}{l}28.20 \\
(5.38)\end{array}$ & $\begin{array}{l}30.20 \\
(5.55)\end{array}$ & $\begin{array}{l}22.60 \\
(4.48)\end{array}$ & $\begin{array}{r}14.40 \\
(3.91)\end{array}$ & $\begin{array}{r}16.38 \\
(3.93)\end{array}$ \\
\hline Eight & $\begin{array}{c}2.00 \\
(1.72)\end{array}$ & $\begin{array}{c}4.00 \\
(2.32)\end{array}$ & $\begin{array}{c}6.40 \\
(2.70)\end{array}$ & $\begin{array}{l}15.80 \\
(4.09)\end{array}$ & $\begin{array}{l}21.80 \\
(4.75)\end{array}$ & $\begin{array}{l}26.80 \\
(5.26)\end{array}$ & $\begin{array}{l}28.80 \\
(5.44)\end{array}$ & $\begin{array}{l}31.20 \\
(5.66)\end{array}$ & $\begin{array}{l}25.40 \\
(5.13)\end{array}$ & $\begin{array}{r}15.00 \\
(3.99)\end{array}$ & $\begin{array}{l}17.72 \\
(4.10)\end{array}$ \\
\hline Nine & $\begin{array}{c}2.80 \\
(1.92)\end{array}$ & $\begin{array}{c}4.20 \\
(2.27)\end{array}$ & $\begin{array}{c}7.80 \\
(2.93)\end{array}$ & $\begin{array}{l}17.00 \\
(4.23)\end{array}$ & $\begin{array}{l}23.80 \\
(4.97)\end{array}$ & $\begin{array}{l}27.60 \\
(5.33)\end{array}$ & $\begin{array}{l}31.60 \\
(5.69)\end{array}$ & $\begin{array}{l}34.80 \\
(5.98)\end{array}$ & $\begin{array}{l}26.40 \\
(5.22)\end{array}$ & $\begin{array}{c}16.80 \\
(4.21)\end{array}$ & $\begin{array}{l}19.28 \\
(4.28)\end{array}$ \\
\hline Ten & $\begin{array}{c}3.00 \\
(1.96)\end{array}$ & $\begin{array}{c}4.80 \\
(2.40)\end{array}$ & $\begin{array}{l}10.20 \\
(3.30)\end{array}$ & $\begin{array}{l}20.20 \\
(4.59)\end{array}$ & $\begin{array}{l}26.80 \\
(5.26)\end{array}$ & $\begin{array}{l}30.20 \\
(5.58)\end{array}$ & $\begin{array}{l}32.20 \\
(5.75)\end{array}$ & $\begin{array}{l}35.60 \\
(6.04)\end{array}$ & $\begin{array}{l}27.60 \\
(5.33)\end{array}$ & $\begin{array}{c}17.20 \\
(4.26)\end{array}$ & $\begin{array}{l}20.78 \\
(4.45)\end{array}$ \\
\hline Mean & $\begin{array}{c}2.40 \\
(1.82)\end{array}$ & $\begin{array}{c}4.05 \\
(2.37)\end{array}$ & $\begin{array}{c}7.60 \\
(2.89)\end{array}$ & $\begin{array}{l}16.40 \\
(4.14)\end{array}$ & $\begin{array}{l}23.05 \\
(4.88)\end{array}$ & $\begin{array}{l}27.40 \\
(5.31)\end{array}$ & $\begin{array}{l}30.20 \\
(5.57)\end{array}$ & $\begin{array}{l}32.95 \\
(5.81)\end{array}$ & $\begin{array}{l}25.50 \\
(5.13)\end{array}$ & $\begin{array}{r}15.85 \\
(4.09)\end{array}$ & - \\
\hline Factors & & & & C.D. & & & $\operatorname{SE}(d)$ & & & $\mathrm{SE}(\mathrm{m})$ & \\
\hline Month & & & & 0.11 & & & 0.05 & & & 0.04 & \\
\hline Combs & & & & 0.18 & & & 0.09 & & & 0.06 & \\
\hline Month $\times$ Combs & & & & 0.36 & & & 0.18 & & & 0.12 & \\
\hline
\end{tabular}


highest number (30.67) of GWM adults were observed in September/. The peak month population was in September $22.01 \%$ of total population of the adults. Fathy et al (2017) observed number of wax moths changed greatly in different months.

Correlation between weather parameters and Greater wax moth (G. mellonella) incidence in stored comb: During both years, the greater wax moth's larvae starts infesting combs from first fortnight of June and progressively to acquire peak in September in 7, 8, 9 and 10 combs/hive. During September, average of weather parameters of both years were recorded as maximum temperature $\left(35.07^{\circ} \mathrm{C}\right)$, minimum temperature $\left(23.63^{\circ} \mathrm{C}\right)$, maximum $\mathrm{RH}(86.72 \%)$, minimum $\mathrm{RH}(51.85 \%)$ and number of bright sunshine hour (7.84 hour). During this period, the weather condition for the development of the greater wax moth was very congenial. In June, the average of different weather parameters recorded as maximum temperature $\left(40.2^{\circ} \mathrm{C}\right)$, minimum temperature $\left(26.2^{\circ} \mathrm{C}\right)$, maximum $\mathrm{RH}(66.45 \%)$, minimum $\mathrm{RH}(35.3 \%)$ and number of bright sunshine hour ( 8.15 hour). Correlation between number of larvae and weather parameters were positive and significant for minimum temperature and maximum $\mathrm{R}$ ), positive and highly significant for minimum $\mathrm{R}$ ). Correlation did not exist between number of larvae and maximum temperature and bright sunshine hours.

The correlation between number of pupae and minimum $\mathrm{RH}$ was positive and highly significant. Correlation did not exist between pupal population and weather parameters such as maximum temperature, minimum temperature, maximum $\mathrm{RH}$ and bright sunshine hours. The correlation between number of adults and weather parameters were be positive and significant for minimum temperature and positive and highly significant for maximum $\mathrm{RH}$, whereas no correlation existed between number of adults and weather

Table 2. Pupal population of Greater wax moth (G. mellonella) during June to October in stored combs during 2016 and 2017

\begin{tabular}{|c|c|c|c|c|c|c|c|c|c|c|c|}
\hline \multirow{3}{*}{$\begin{array}{l}\text { Number of combs in } \\
\text { hive body }\end{array}$} & \multicolumn{10}{|c|}{ Cumulative number of pupae/comb during different months of 2016} & \multirow[t]{3}{*}{ Mean } \\
\hline & \multicolumn{2}{|c|}{ June } & \multicolumn{2}{|c|}{ July } & \multicolumn{2}{|c|}{ August } & \multicolumn{2}{|c|}{ September } & \multicolumn{2}{|c|}{ October } & \\
\hline & 15.6.16 & 30.6 .16 & 15.7.16 & 30.7 .16 & 15.8.16 & 30.8 .16 & 15.9.16 & 30.9 .16 & 15.10.16 & 30.10 .16 & \\
\hline Seven & $\begin{array}{c}0.20 \\
(1.08)\end{array}$ & $\begin{array}{l}1.40 \\
(1.54)\end{array}$ & $\begin{array}{c}2.80 \\
(1.93)\end{array}$ & $\begin{array}{l}8.20 \\
(3.02)\end{array}$ & $\begin{array}{l}12.80 \\
(3.70)\end{array}$ & $\begin{array}{l}21.40 \\
(4.68)\end{array}$ & $\begin{array}{l}25.80 \\
(5.15)\end{array}$ & $\begin{array}{l}26.80 \\
(5.25)\end{array}$ & $\begin{array}{l}19.60 \\
(4.52)\end{array}$ & $\begin{array}{c}9.40 \\
(3.21)\end{array}$ & $\begin{array}{l}12.86 \\
(3.41)\end{array}$ \\
\hline Eight & $\begin{array}{c}0.40 \\
(1.14)\end{array}$ & $\begin{array}{l}2.20 \\
(1.76)\end{array}$ & $\begin{array}{c}3.00 \\
(1.99)\end{array}$ & $\begin{array}{l}12.00 \\
(3.59)\end{array}$ & $\begin{array}{l}19.20 \\
(4.46)\end{array}$ & $\begin{array}{l}23.40 \\
(4.92)\end{array}$ & $\begin{array}{l}26.60 \\
(5.25)\end{array}$ & $\begin{array}{l}27.40 \\
(5.32)\end{array}$ & $\begin{array}{l}20.00 \\
(4.57)\end{array}$ & $\begin{array}{l}10.40 \\
(3.36)\end{array}$ & $\begin{array}{l}14.44 \\
(3.63)\end{array}$ \\
\hline Nine & $\begin{array}{c}1.00 \\
(1.34)\end{array}$ & $\begin{array}{l}3.80 \\
(2.18)\end{array}$ & $\begin{array}{c}4.60 \\
(2.36)\end{array}$ & $\begin{array}{l}14.60 \\
(3.94)\end{array}$ & $\begin{array}{l}20.80 \\
(4.66)\end{array}$ & $\begin{array}{l}24.40 \\
(5.03)\end{array}$ & $\begin{array}{l}30.60 \\
(5.60)\end{array}$ & $\begin{array}{l}33.00 \\
(5.82)\end{array}$ & $\begin{array}{l}22.80 \\
(4.87)\end{array}$ & $\begin{array}{l}10.80 \\
(3.42)\end{array}$ & $\begin{array}{l}16.64 \\
(3.92)\end{array}$ \\
\hline Ten & $\begin{array}{c}1.40 \\
(1.54)\end{array}$ & $\begin{array}{c}4.80 \\
(2.39)\end{array}$ & $\begin{array}{c}6.00 \\
(2.64)\end{array}$ & $\begin{array}{l}15.20 \\
(4.01)\end{array}$ & $\begin{array}{l}23.00 \\
(4.89)\end{array}$ & $\begin{array}{l}27.40 \\
(5.32)\end{array}$ & $\begin{array}{l}32.20 \\
(5.75)\end{array}$ & $\begin{array}{l}34.80 \\
(5.98)\end{array}$ & $\begin{array}{l}23.80 \\
(4.97)\end{array}$ & $\begin{array}{l}12.80 \\
(3.71)\end{array}$ & $\begin{array}{l}18.14 \\
(4.12)\end{array}$ \\
\hline Mean & $\begin{array}{c}0.75 \\
(1.27)\end{array}$ & $\begin{array}{l}3.05 \\
(1.97)\end{array}$ & $\begin{array}{c}4.10 \\
(2.23)\end{array}$ & $\begin{array}{l}12.50 \\
(3.64)\end{array}$ & $\begin{array}{l}18.95 \\
(4.43)\end{array}$ & $\begin{array}{l}24.15 \\
(4.99)\end{array}$ & $\begin{array}{l}28.20 \\
(5.44)\end{array}$ & $\begin{array}{l}30.50 \\
(5.59)\end{array}$ & $\begin{array}{l}21.55 \\
(4.73)\end{array}$ & $\begin{array}{l}10.85 \\
(3.43)\end{array}$ & - \\
\hline Factors & & & & C.D. & & & $\operatorname{SE}(d)$ & & & $\mathrm{SE}(\mathrm{m})$ & \\
\hline Month & & & & 0.12 & & & 0.06 & & & 0.04 & \\
\hline Combs & & & & 0.20 & & & 0.10 & & & 0.07 & \\
\hline \multirow[t]{2}{*}{ Month $\times$ Combs } & & & & 0.40 & & & 0.20 & & & 0.14 & \\
\hline & \multicolumn{10}{|c|}{ Cumulative number of pupae/comb during different months of 2017} & Mean \\
\hline Seven & $\begin{array}{c}0.60 \\
(1.22)\end{array}$ & $\begin{array}{c}1.80 \\
(1.65)\end{array}$ & $\begin{array}{c}3.00 \\
(1.97)\end{array}$ & $\begin{array}{l}8.80 \\
(3.11)\end{array}$ & $\begin{array}{l}13.60 \\
(3.80)\end{array}$ & $\begin{array}{l}21.00 \\
(5.64)\end{array}$ & $\begin{array}{l}26.20 \\
(5.20)\end{array}$ & $\begin{array}{l}27.60 \\
(5.32)\end{array}$ & $\begin{array}{l}20.00 \\
(4.56)\end{array}$ & $\begin{array}{l}10.40 \\
(3.36)\end{array}$ & $\begin{array}{l}13.30 \\
(3.48)\end{array}$ \\
\hline Eight & $\begin{array}{c}1.00 \\
(1.41)\end{array}$ & $\begin{array}{c}2.00 \\
(1.70)\end{array}$ & $\begin{array}{c}3.40 \\
(2.08)\end{array}$ & $\begin{array}{l}12.80 \\
(3.70)\end{array}$ & $\begin{array}{l}19.80 \\
(4.52)\end{array}$ & $\begin{array}{l}24.80 \\
(5.06)\end{array}$ & $\begin{array}{l}27.00 \\
(5.28)\end{array}$ & $\begin{array}{l}28.40 \\
(5.41)\end{array}$ & $\begin{array}{l}21.20 \\
(4.71)\end{array}$ & $\begin{array}{l}11.20 \\
(3.49)\end{array}$ & $\begin{array}{l}15.16 \\
(3.74)\end{array}$ \\
\hline Nine & $\begin{array}{c}1.20 \\
(1.39)\end{array}$ & $\begin{array}{c}4.00 \\
(2.23)\end{array}$ & $\begin{array}{c}4.80 \\
(2.40)\end{array}$ & $\begin{array}{l}15.00 \\
(3.99)\end{array}$ & $\begin{array}{l}21.60 \\
(4.75)\end{array}$ & $\begin{array}{l}25.60 \\
(5.15)\end{array}$ & $\begin{array}{l}30.20 \\
(5.57)\end{array}$ & $\begin{array}{l}33.20 \\
(5.84)\end{array}$ & $\begin{array}{l}23.80 \\
(4.97)\end{array}$ & $\begin{array}{l}12.40 \\
(3.65)\end{array}$ & $\begin{array}{l}17.18 \\
(3.99)\end{array}$ \\
\hline Ten & $\begin{array}{c}1.40 \\
(1.47)\end{array}$ & $\begin{array}{l}5.00 \\
(2.44)\end{array}$ & $\begin{array}{c}6.40 \\
(2.71)\end{array}$ & $\begin{array}{l}17.20 \\
(4.25)\end{array}$ & $\begin{array}{l}25.00 \\
(5.09)\end{array}$ & $\begin{array}{l}27.20 \\
(5.30)\end{array}$ & $\begin{array}{l}32.60 \\
(5.79)\end{array}$ & $\begin{array}{l}34.40 \\
(5.94)\end{array}$ & $\begin{array}{l}24.40 \\
(5.03)\end{array}$ & $\begin{array}{l}13.60 \\
(3.81)\end{array}$ & $\begin{array}{l}18.72 \\
(4.18)\end{array}$ \\
\hline Mean & $\begin{array}{c}1.05 \\
(1.37)\end{array}$ & $\begin{array}{c}3.20 \\
(2.01)\end{array}$ & $\begin{array}{c}4.40 \\
(2.29)\end{array}$ & $\begin{array}{l}13.45 \\
(3.76)\end{array}$ & $\begin{array}{l}20.00 \\
(4.54)\end{array}$ & $\begin{array}{l}24.65 \\
(5.04)\end{array}$ & $\begin{array}{l}29.00 \\
(5.46)\end{array}$ & $\begin{array}{l}30.90 \\
(5.63)\end{array}$ & $\begin{array}{l}22.35 \\
(4.82)\end{array}$ & $\begin{array}{l}11.90 \\
(3.58)\end{array}$ & - \\
\hline Factors & & & & C.D. & & & $\mathrm{SE}(\mathrm{d})$ & & & $\mathrm{SE}(\mathrm{m})$ & \\
\hline Month & & & & 0.13 & & & 0.06 & & & 0.04 & \\
\hline Combs & & & & 0.20 & & & 0.10 & & & 0.07 & \\
\hline Month $\times$ Combs & & & & 0.41 & & & 0.20 & & & 0.14 & \\
\hline
\end{tabular}


parameters as maximum temperature, minimum $\mathrm{RH}$ and bright sunshine hour. In 8 combs stored per hive, the correlation between number of larvae and weather parameters were positive and highly significant for minimum temperature, maximum $\mathrm{RH}$, minimum $\mathrm{RH}$ and negative and significant for bright sunshine hours. Correlation did not exist between larvae and maximum temperature. The correlation between number of pupae and weather parameters were positive and highly significant for minimum temperature), maximum $\mathrm{R}$ ), minimum $\mathrm{RH}$ and negative and significant for bright sunshine hour. Correlation did not exist between number of pupae and maximum temperature. The correlation between number of adults and weather parameters were positive and highly significant for minimum temperature, minimum $\mathrm{RH}$, positive and significant for maximum $\mathrm{RH}$, whereas negative and significant for bright sunshine hour. No correlation existed between adult and maximum temperature. In 9 combs stored per hive. The correlation between number of larvae and weather parameters were found positive and highly significant for maximum temperature, minimum $\mathrm{RH}$ and positive and significant for maximum $\mathrm{RH}$. The correlation between number of pupae and weather parameters were positive and highly significant for minimum $\mathrm{RH}$ and positive and significant for minimum temperature. No correlation existed between number of pupae and weather parameters for maximum temperature, maximum $\mathrm{RH}$ and bright sunshine hours. In 10 frame stored in hive body, the correlation between number of larvae and weather parameters was found positive and highly significant for minimum $\mathrm{RH}(r=0.311)$ but no correlation existed between larval and weather parameters. The correlation between number of pupae and weather parameters were

Table 3. Seasonal incidence of Greater wax moth (G. mellonella) adult during June to October in stored combs during 2016 and 2017

\begin{tabular}{|c|c|c|c|c|c|c|c|c|c|c|c|}
\hline \multirow{3}{*}{$\begin{array}{l}\text { Number of combs in } \\
\text { hive body }\end{array}$} & \multicolumn{10}{|c|}{ Cumulative number of adults/comb during different months of 2016} & \multirow[t]{3}{*}{ Mean } \\
\hline & \multicolumn{2}{|c|}{ June } & \multicolumn{2}{|c|}{ July } & \multicolumn{2}{|c|}{ August } & \multicolumn{2}{|c|}{ September } & \multicolumn{2}{|c|}{ October } & \\
\hline & 15.6.16 & 30.6 .16 & 15.7.16 & 30.7 .16 & 15.8.16 & 30.8 .16 & 15.9.16 & 30.9 .16 & 15.10 .16 & 30.10 .16 & \\
\hline Seven & $\begin{array}{c}0.00 \\
(1.00)\end{array}$ & $\begin{array}{c}1.00 \\
(1.37)\end{array}$ & $\begin{array}{c}1.40 \\
(1.53)\end{array}$ & $\begin{array}{c}6.00 \\
(2.63)\end{array}$ & $\begin{array}{l}11.60 \\
(3.53)\end{array}$ & $\begin{array}{l}17.60 \\
(4.29)\end{array}$ & $\begin{array}{l}23.40 \\
(4.93)\end{array}$ & $\begin{array}{l}26.00 \\
(5.18)\end{array}$ & $\begin{array}{l}15.20 \\
(4.01)\end{array}$ & $\begin{array}{c}8.20 \\
(3.02)\end{array}$ & $\begin{array}{l}11.04 \\
(3.15)\end{array}$ \\
\hline Eight & $\begin{array}{c}0.40 \\
(1.16)\end{array}$ & $\begin{array}{c}1.40 \\
(1.54)\end{array}$ & $\begin{array}{c}2.80 \\
(1.94)\end{array}$ & $\begin{array}{c}9.40 \\
(3.21)\end{array}$ & $\begin{array}{l}16.80 \\
(4.21)\end{array}$ & $\begin{array}{l}20.40 \\
(4.62)\end{array}$ & $\begin{array}{l}24.00 \\
(4.99)\end{array}$ & $\begin{array}{l}27.00 \\
(5.27)\end{array}$ & $\begin{array}{l}16.60 \\
(4.18)\end{array}$ & $\begin{array}{c}8.80 \\
(3.12)\end{array}$ & $\begin{array}{l}12.76 \\
(3.42)\end{array}$ \\
\hline Nine & $\begin{array}{c}0.80 \\
(1.29)\end{array}$ & $\begin{array}{c}3.00 \\
(1.98)\end{array}$ & $\begin{array}{c}4.00 \\
(2.23)\end{array}$ & $\begin{array}{l}11.80 \\
(3.56)\end{array}$ & $\begin{array}{l}18.80 \\
(4.44)\end{array}$ & $\begin{array}{l}22.80 \\
(4.87)\end{array}$ & $\begin{array}{l}25.20 \\
(5.11)\end{array}$ & $\begin{array}{l}30.20 \\
(5.56)\end{array}$ & $\begin{array}{l}19.40 \\
(4.50)\end{array}$ & $\begin{array}{c}9.80 \\
(3.28)\end{array}$ & $\begin{array}{l}14.58 \\
(4.68)\end{array}$ \\
\hline Ten & $\begin{array}{c}1.20 \\
(1.47)\end{array}$ & $\begin{array}{c}3.20 \\
(2.03)\end{array}$ & $\begin{array}{c}4.40 \\
(2.31)\end{array}$ & $\begin{array}{l}14.60 \\
(3.94)\end{array}$ & $\begin{array}{l}19.80 \\
(4.55)\end{array}$ & $\begin{array}{l}23.20 \\
(4.91)\end{array}$ & $\begin{array}{l}27.80 \\
(5.36)\end{array}$ & $\begin{array}{l}32.40 \\
(5.77)\end{array}$ & $\begin{array}{l}19.60 \\
(4.51)\end{array}$ & $\begin{array}{l}10.40 \\
(3.37)\end{array}$ & $\begin{array}{l}15.66 \\
(3.82)\end{array}$ \\
\hline Mean & $\begin{array}{c}0.60 \\
(1.23)\end{array}$ & $\begin{array}{c}2.15 \\
(1.73)\end{array}$ & $\begin{array}{c}3.15 \\
(2.00)\end{array}$ & $\begin{array}{l}10.45 \\
(3.34)\end{array}$ & $\begin{array}{l}16.75 \\
(4.18)\end{array}$ & $\begin{array}{l}21.00 \\
(4.67)\end{array}$ & $\begin{array}{l}25.10 \\
(5.09)\end{array}$ & $\begin{array}{l}28.90 \\
(5.45)\end{array}$ & $\begin{array}{l}17.70 \\
(4.30)\end{array}$ & $\begin{array}{c}9.30 \\
(3.20)\end{array}$ & - \\
\hline Factors & & & & C.D. & & & $\operatorname{SE}(d)$ & & & $\mathrm{SE}(\mathrm{m})$ & \\
\hline Month & & & & 0.11 & & & 0.06 & & & 0.04 & \\
\hline Combs & & & & 0.18 & & & 0.09 & & & 0.06 & \\
\hline \multirow[t]{2}{*}{ Month $\times$ Combs } & & & & 0.36 & & & 0.18 & & & 0.13 & \\
\hline & \multicolumn{10}{|c|}{ Cumulative number of adults/comb during different months of 2017} & Mean \\
\hline Seven & $\begin{array}{c}0.00 \\
(1.00)\end{array}$ & $\begin{array}{c}1.60 \\
(1.61)\end{array}$ & $\begin{array}{c}2.00 \\
(1.71)\end{array}$ & $\begin{array}{c}6.40 \\
(2.71)\end{array}$ & $\begin{array}{l}12.60 \\
(3.68)\end{array}$ & $\begin{array}{l}18.80 \\
(4.42)\end{array}$ & $\begin{array}{l}25.00 \\
(5.09)\end{array}$ & $\begin{array}{l}27.00 \\
(5.27)\end{array}$ & $\begin{array}{l}16.80 \\
(4.19)\end{array}$ & $\begin{array}{c}9.00 \\
(3.14)\end{array}$ & $\begin{array}{l}11.92 \\
(3.28)\end{array}$ \\
\hline Eight & $\begin{array}{c}0.60 \\
(1.24)\end{array}$ & $\begin{array}{c}1.80 \\
(1.65)\end{array}$ & $\begin{array}{c}2.60 \\
(1.89)\end{array}$ & $\begin{array}{c}9.60 \\
(3.24)\end{array}$ & $\begin{array}{l}17.80 \\
(4.31)\end{array}$ & $\begin{array}{l}20.80 \\
(4.66)\end{array}$ & $\begin{array}{l}25.40 \\
(5.13)\end{array}$ & $\begin{array}{l}27.80 \\
(5.35)\end{array}$ & $\begin{array}{l}17.40 \\
(4.27)\end{array}$ & $\begin{array}{c}9.20 \\
(3.19)\end{array}$ & $\begin{array}{l}13.30 \\
(3.49)\end{array}$ \\
\hline Nine & $\begin{array}{c}1.00 \\
(1.34)\end{array}$ & $\begin{array}{c}3.40 \\
(2.09)\end{array}$ & $\begin{array}{c}4.20 \\
(2.27)\end{array}$ & $\begin{array}{l}12.20 \\
(3.62)\end{array}$ & $\begin{array}{l}20.40 \\
(4.62)\end{array}$ & $\begin{array}{l}23.80 \\
(4.97)\end{array}$ & $\begin{array}{l}26.00 \\
(5.18)\end{array}$ & $\begin{array}{l}31.60 \\
(5.70)\end{array}$ & $\begin{array}{l}20.80 \\
(4.65)\end{array}$ & $\begin{array}{l}10.80 \\
(3.42)\end{array}$ & $\begin{array}{l}15.42 \\
(3.79)\end{array}$ \\
\hline Ten & $\begin{array}{c}1.00 \\
(1.39)\end{array}$ & $\begin{array}{c}4.20 \\
(2.26)\end{array}$ & $\begin{array}{c}4.80 \\
(2.40)\end{array}$ & $\begin{array}{l}15.80 \\
(4.08)\end{array}$ & $\begin{array}{l}21.40 \\
(4.73)\end{array}$ & $\begin{array}{l}24.00 \\
(4.99)\end{array}$ & $\begin{array}{l}28.40 \\
(5.41)\end{array}$ & $\begin{array}{l}32.40 \\
(5.77)\end{array}$ & $\begin{array}{l}20.80 \\
(4.64)\end{array}$ & $\begin{array}{l}11.00 \\
(3.45)\end{array}$ & $\begin{array}{l}16.38 \\
(3.91)\end{array}$ \\
\hline Mean & $\begin{array}{c}0.65 \\
(1.24)\end{array}$ & $\begin{array}{c}2.75 \\
(1.90)\end{array}$ & $\begin{array}{c}3.40 \\
(2.07)\end{array}$ & $\begin{array}{l}11.00 \\
(3.41)\end{array}$ & $\begin{array}{l}18.05 \\
(4.33)\end{array}$ & $\begin{array}{l}21.85 \\
(3.76)\end{array}$ & $\begin{array}{l}26.20 \\
(5.20)\end{array}$ & $\begin{array}{l}29.70 \\
(5.52)\end{array}$ & $\begin{array}{l}18.95 \\
(4.44)\end{array}$ & $\begin{array}{l}10.00 \\
(3.30)\end{array}$ & - \\
\hline Factors & & & & C.D. & & & $\operatorname{SE}(d)$ & & & $\mathrm{SE}(\mathrm{m})$ & \\
\hline Month & & & & 0.12 & & & 0.06 & & & 0.04 & \\
\hline Combs & & & & 0.19 & & & 0.10 & & & 0.07 & \\
\hline Month $\times$ Combs & & & & 0.39 & & & 0.20 & & & 0.14 & \\
\hline
\end{tabular}


Table 4. Correlation between weather parameters and greater wax moth, Galleria mellonella in stored comb (without bees) with different strength during both years

\begin{tabular}{|c|c|c|c|c|c|c|c|c|c|c|c|c|}
\hline \multirow{3}{*}{$\begin{array}{l}\text { Weather } \\
\text { parameters }\end{array}$} & \multicolumn{12}{|c|}{ Number of empty combs group/ hive body/ chamber during both years } \\
\hline & \multicolumn{3}{|c|}{7 frame } & \multicolumn{3}{|c|}{8 frame } & \multicolumn{3}{|c|}{9 frame } & \multicolumn{3}{|c|}{10 frame } \\
\hline & Larvae & Pupae & Adults & Larvae & Pupae & Adults & Larvae & Pupae & Adults & Larvae & Pupae & Adults \\
\hline Max. temp $\left({ }^{\circ} \mathrm{C}\right)$ & $0.138^{*}$ & 0.122 & 0.119 & 0.170 & 0.162 & 0.194 & 0.214 & 0.186 & 0.146 & 0.192 & $0.143^{*}$ & 0.214 \\
\hline Min. temp. $\left({ }^{\circ} \mathrm{C}\right)$ & 0.252 & 0.237 & 0.217 & $0.327^{\star *}$ & $0.289^{* *}$ & $0.377^{* *}$ & $0.380^{* *}$ & $0.378^{* *}$ & $0.332^{* *}$ & $0.353^{*}$ & $0.251^{*}$ & $0.389^{*}$ \\
\hline Max. RH (\%) & $0.356^{* *}$ & $0.373^{* *}$ & $0.265^{*}$ & $0.438^{* *}$ & $0.343^{* *}$ & $0.455^{\star *}$ & $0.462^{* *}$ & $0.469^{*}$ & $0.386^{* *}$ & $0.468^{* *}$ & $0.350^{* *}$ & $0.451^{* \star}$ \\
\hline Min. RH (\%) & 0.223 & 0.207 & $0.121^{*}$ & $0.301^{* *}$ & 0.200 & $0.257^{*}$ & $0.264^{\star *}$ & $0.270^{*}$ & 0.224 & $0.311^{*}$ & 0.196 & 0.194 \\
\hline Bright sunshine (hr) & -0.083 & -0.09 & -0.076 & -0.138 & -0.164 & $-0.189^{*}$ & $-0.164^{*}$ & -0.248 & -0.236 & -0.149 & -0.046 & -0.112 \\
\hline
\end{tabular}

found positive and highly significant for minimum $\mathrm{RH}$. No correlation existed between number of pupae and maximum temperature, minimum temperature, maximum $\mathrm{RH}$ and bright sunshine hour. The correlation between number of adults and weather parameters were positive and significant for minimum R). Correlation also did not exist between adult and weather parameters such as maximum temperature, minimum temperature, $\mathrm{RH}$ and number of bright sunshine hour. Mandal and Vishwakarma (2016) reported positive and significant in maximum temperature, minimum temperature and minimum relative humidity with the larval population of GWM per hive, whereas the negative non-significant correlation with the maximum relative humidity and positive non-significant correlation with rainfall. Therefore, it is concluded that the GWM population were positively correlated with temperature and minimum relative humidity.

\section{CONCLUSIONS}

During both the years, larval, pupal and adult population in stored combs increased with the passage of time from June to September. However, in October, the cumulative number of larvae, pupae and adults decreased. Larval, pupal and adult population was highest when ten combs were stored followed by 9,8 and 7 combs. Correlation studies between number of greater wax moth population in stored combs related to different frame strength with the different weather parameters and showed significant positive correlation with maximum $\mathrm{RH}$ and maximum temperature, whereas no significant correlation existed with minimum temperature, minimum $\mathrm{RH}$ and sunshine hours during both the years.

\section{REFERENCES}

Ellis AM and Hayes G 2009. Assessing the efficacy of a product containing Bacillus thuringiensis applied to honey bee (Hymenoptera: Apidae) foundation as a control for Galleria mellonella (Lepidoptera: Pyralidae). Journal of Entomological Science 44(2): 158-163.

Ellis JD, Graham JR and Mortensen A 2013 Standard methods for wax moth research. Journal of Apicultural Research 52(1): 117.

Fathy DM, Fathy HM, Mansour HM and Ziedan MAR 2017. Activity of the greater wax moth Galleria mellonella L. and the lesser wax moth Achroia grisella F. in apiary and storage in Kafr El-Sheikh Province. Journal of Plant Protection and Pathology 8(10): 497500.

Gillard G 2009. My friend, the wax moth. American Bee Journal 149(6): 559-562.

Kumari S and Jha MM 2013 Efficacy of non-chemical method in management of wax moth infestation of Apis mellifera. Bulletin of Pure and Applied Sciences 32(1): 37-44.

Mandal S and Vishvakarma R 2016 Population dynamics of greater wax moth (Galleria mellonella L.) infesting Apis mellifera L. combs during dearth period. The Ecoscan 9: 93-98 